\title{
Right-Angle Shaped Elements as Dual-Band Metamaterial Absorber in Terahertz
}

\author{
Salman DANIEL ${ }^{1 *}$ and Prince BAWUAH ${ }^{2}$ \\ ${ }^{1}$ Institute of Photonics, University of Eastern Finland, Joensuu FI-80101, Finland \\ ${ }^{2}$ Department of Chemical Engineering and Biotechnology, University of Cambridge, Cambridge CB3 0AS, \\ United Kingdom \\ ${ }^{*}$ Corresponding author: Salman DANIEL_Ｅ-mail: salman.daniel@hotmail.com and pb612@cam.ac.uk
}

\begin{abstract}
Metamaterial absorbers display potential applications in the field of photonics and have been investigated extensively during the last decade. We propose a dual-band resonant metamaterial absorber with right-angle shaped elements (RAEs) in the terahertz range based on numerical simulations. The absorber remains insensitive to a wide range of incidence angles $\left(0^{\circ}-70^{\circ}\right)$ by showing a minimum absorbance of $\sim 80 \%$ at $70^{\circ}$. Furthermore, the proposed absorber is highly independent on any state of polarization of the incidence electromagnetic wave due to the high absorbance, i.e., greater than $80 \%$, recorded for the considered polarization states. To further comprehend the slight variations in absorbance as a function of change in the angle of incidence, the impedance of the structure has been critically examined. The metamaterial absorber is simple in design, and we provide a possible path of fabrication.
\end{abstract}

Keywords: Metamaterial; absorbance; photonics devices; terahertz

Citation: Salman DANIEL and Prince BAWUAH, "Right-Angle Shaped Elements as Dual-Band Metamaterial Absorber in Terahertz," Photonic Sensors, 2020, 10(3): 233-241.

\section{Introduction}

The recent surge in the study of terahertz-based metamaterials is due to their unusual electromagnetic (EM) behavior [1, 2] and hence unique applications [3]. The unconventional properties of metamaterials emerge due to their negative refractive indices, which is impossible to be observed in naturally existing materials [4-6]. In recent time, metamaterial absorbers are introduced in different regions of the EM spectrum, e.g., infrared [7], visible [8], and microwave ranges [9]. Typically, a metamaterial absorber should either give high absorbance at a specific frequency or at a given range of frequencies [10]. Therefore, both reflection and transmission should be minimum at that specific or range of frequencies. A minimum value for the reflection and the transmission can be achieved by impedance matching approach of the structure [11]. A typical expectation of an ideal absorber is its ability to render $100 \%$ absorbance at a wide range of both incidence and polarization angles of an incidence EM wave [12]. Many metamaterial absorbers with different designs and performances, for example, electric resonators [13], arrays [14], thin wire-crossed [15], crossed-shaped for bio sensing [16], bilayer [17], circular-sectors [18], metal groove features [19], slotted sectors [20],

Received: 6 May 2019 / Revised: 21 August 2019

(C) The Author(s) 2019. This article is published with open access at Springerlink.com

DOI: $10.1007 / \mathrm{s} 13320-019-0573-6$

Article type: Regular 
ultrathin [21], dual-band [22-24], and triple-band absorbers [25, 26], have been studied. In addition, numerous methods are applied to control or vary the absorbance of the metamaterial device, e.g., those in [8, 27-31]. Generally, from the application point of view, the frequency region of interest lies in terahertz $(\mathrm{THz})$ range due to the difficulty to get naturally existing resonant absorbers in this part of the EM spectrum [32]. Moreover, resonant metamaterial absorbers in the $\mathrm{THz}$ range are relatively easy to be manufactured due to their relatively large sizes in the micrometer range. The applications of resonant metamaterial absorbers include civilian and military products in the form of thermal detectors or coating layers [3, 33, 34].

One of the challenges for designing or fabricating a metamaterial absorber is to keep a relatively high absorbance with respect to the change in incidence as well as polarization angles within a certain range of frequencies [35]. Herein, we propose a design of a dual-band metamaterial absorber in the terahertz range based on finite element method simulations. The absorbance approaches unity at two resonant frequencies of $2.71 \mathrm{THz}$ and $4.17 \mathrm{THz}$. The structure provides a higher absorbance $(\sim 80 \%)$ in a wide range of incidence angles $\left(0^{\circ}-70^{\circ}\right)$ for both TE and TM modes than some earlier reported dual-band absorbers [36-40]. Moreover, the absorber displays a relatively high insensitivity (with absorbance greater than $80 \%$ ) at all angles of polarization between $0^{\circ}$ and $90^{\circ}$ of the incidence EM wave. However, the slight variations observed in both the absorbance and the resonant frequencies with respect to the angle of incidence have been analyzed by adopting the impedance matching approach. The proposed absorber is simple in design which consists of right-angle shaped elements at a top surface and a metallic layer of aluminum (Al) at the bottom. The right-angle shaped elements (RAEs) assist to enhance the charge storing ability of the structure by aligning a large amount of charges in the direction of the field, which eventually induces high absorption within the structure. The two metallic parts of the device are separated by a layer of dielectric $\mathrm{TiO}_{2}$. Finally, the use of $\mathrm{Al}$ and $\mathrm{TiO}_{2}$ in the design indicates an easy route of manufacturing the absorber. This is due to the fact that both $\mathrm{Al}$ and $\mathrm{TiO}_{2}$ are commonly used materials in most fabrication processes.

\section{Design and theory}

A design of the metamaterial absorber with its optimized parameters is shown in Fig. 1. Four RAEs, which appear in a yellow color at the top, are made of aluminum (Al) with an optimized thickness of $100 \mathrm{~nm}$. The RAEs are aligned in such a way that the state of polarization of an incidence $\mathrm{THz}$ wave has a minimum effect on the performance of the absorber. A layer of $\mathrm{Al}$ with the thickness of $200 \mathrm{~nm}$ is placed at the bottom of the device to avoid any possible transmission of energy through the structure. The two metallic parts, RAEs and Al layer, are separated by a $2 \mu \mathrm{m}$ layer of a dielectric $\mathrm{TiO}_{2}$. A plane wave with a wave vector $\mathbf{k}$ is incident on the structure in a $x z$-plane of incidence at an angle of $\Omega . \Omega$ is the angle

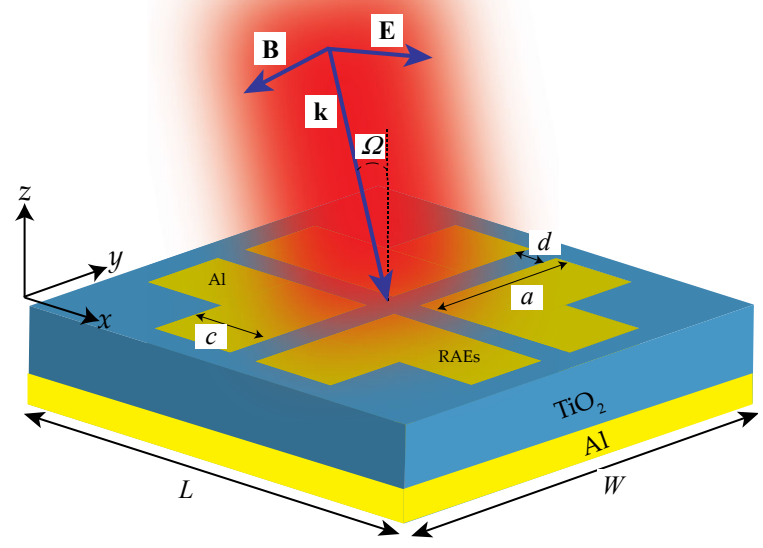

Fig. 1 Schematic diagram of the designed metamaterial absorber. The optimized parameters of RAEs are the following; $a=15 \mu \mathrm{m}, c=7 \mu \mathrm{m}$, and $d=1 \mu \mathrm{m}$. The thickness of the whole device is $2.3 \mu \mathrm{m}$. A plane wave with a wave vector $\mathbf{k}$ is propagating along the negative $z$-axis incidents at the structure with an incidence angle of $\Omega$. $\mathbf{E}$ and $\mathbf{B}$ are electric and magnetic fields, respectively, which are perpendicular to $\mathbf{k}$. The thicknesses of $\mathrm{TiO}_{2}$ and $\mathrm{Al}$ layers are $2 \mu \mathrm{m}$ and $200 \mathrm{~nm}$, respectively. 
between $\mathbf{k}$ and the normal to the surface. $\mathbf{E}$ and $\mathbf{B}$ represent electric and magnetic fields, respectively. The dimension of the device, length $(L) \times$ width $(W)$, is $36 \mu \mathrm{m} \times 36 \mu \mathrm{m}$. The used materials, $\mathrm{Al}$ and $\mathrm{TiO}_{2}$, have large values of the imaginary part of refractive index in the terahertz region [41, 42], which is an indication of high absorbance. The material properties, e.g., optical permittivity, of $\mathrm{Al}$ and $\mathrm{TiO}_{2}$ are used from the Lorentz-Drude model [43] and DeVore 1951 [44], respectively.

In principle, for a perfect absorber, reflection and transmission should be zero. Therefore, the absorbance reaches the maximum at a resonance frequency of interest, e.g., $A=1-R-T$, where $A, R$, and $T$ represent absorbance, reflection, and transmission, respectively. The $R$ becomes zero if the impedance of the structure $Z$ is equal to the impedance of the free space. The transmission $T$ needs to be minimum to maximize the absorbance at the resonant frequency. Hence, to set $T$ to zero, the design makes use of the second layer of $\mathrm{Al}$ with the thickness greater than the skin depth $(\sim 35 \mathrm{~nm})$ of the incident $\mathrm{THz}$ radiation. The impedance $Z$ of the structure can be calculated from the S-parameters [45]. The impedance is extracted from the calculated scattering parameters by using the following expression:

$$
Z(\omega)=\left[\left(1+S_{11}\right)^{2} /\left(1-S_{11}\right)^{2}\right]^{1 / 2}
$$

where $S_{11}$ is the reflection coefficient, and $\omega$ is the angular frequency [22]. In Fig. 2, the real and the imaginary parts of the relative impedance are plotted as a function of frequency $(2 \mathrm{THz}-5 \mathrm{THz})$. The incidence EM wave is polarized along the $y$-direction. In the real part, two peaks appear at resonant frequencies of $2.71 \mathrm{THz}$ and $4.17 \mathrm{THz}$. Similarly, a rapid change is observed in the imaginary part at the same values of frequency. At the resonant frequency, the real part approaches a maximum value, for example, 3 and 1 for $2.71 \mathrm{THz}$ and $4.17 \mathrm{THz}$, respectively. For an ideal absorber, the real part of $Z$ should be 1 at a resonant frequency.
The deviation of impedance value at $2.71 \mathrm{THz}$ from an ideal value 1 affects the performance of the absorber within a range of incidence angles, which will be discussed later. The imaginary part of $Z$ approaches zero at a resonant frequency.

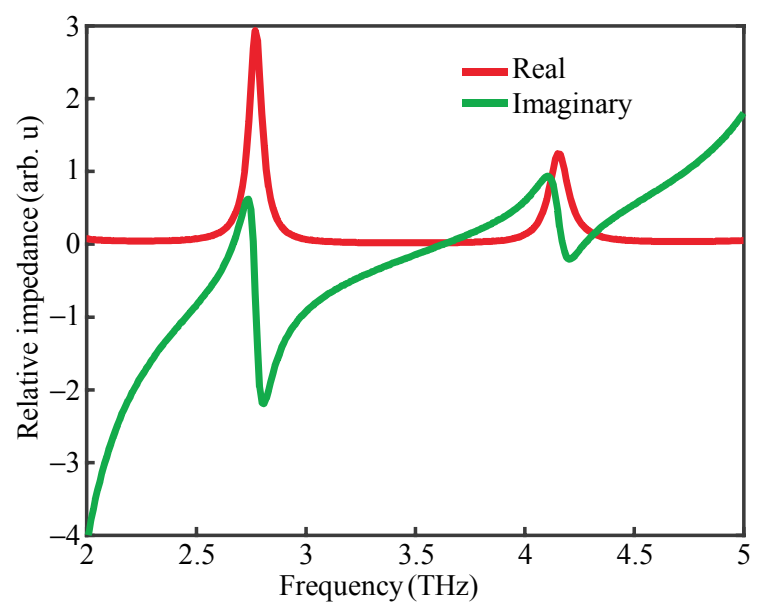

Fig. 2 Plot of the real and imaginary parts of the relative impedance showing dual resonances at $2.71 \mathrm{THz}$ and $4.15 \mathrm{THz}$.

\section{Simulations and results}

A whole unit cell, as shown in Fig. 1, is simulated by employing a finite element method (COMSOL Multiphysics). We apply a periodic port at the top of the structure in the $x y$-plane for the excitation of the EM plane wave that is propagating in the negative $z$-axis direction (see the axis in Fig. 1). The angle of incidence with respect to the normal of the surface is $\Omega$ and the electric field $\mathbf{E}$ is oriented either in the $x$ - or $y$-axis direction. The used conductivity value of $\mathrm{Al}$ is $37 \times 10^{6} \mathrm{~S} / \mathrm{m}$. The $S$-parameters $S_{11}{ }^{2}$ and $S_{21}{ }^{2}$, which are reflection and transmission coefficients, respectively, are calculated. The transmission coefficient $S_{21}{ }^{2}$ is zero due to the metallic layer that is placed at the bottom of the structure. Reflection as a function of frequency in a range of $2 \mathrm{THz}-5 \mathrm{THz}$ is plotted in Fig. 3. At two resonant frequencies, $2.71 \mathrm{THz}$ and $4.17 \mathrm{THz}$, reflection tends to approach zero. For a normal incidence angle $\left(\Omega=0^{\circ}\right)$, the reflection values are 0.06 and 0 for $2.71 \mathrm{THz}$ and $4.17 \mathrm{THz}$, respectively. A small difference in $R$ is due to the impedance mismatch at this value of $\Omega$, and we will 
discuss it later. Nevertheless, Figure 3 already indicates an impressive response of the designed dual-band metamaterial in the $\mathrm{THz}$ region.

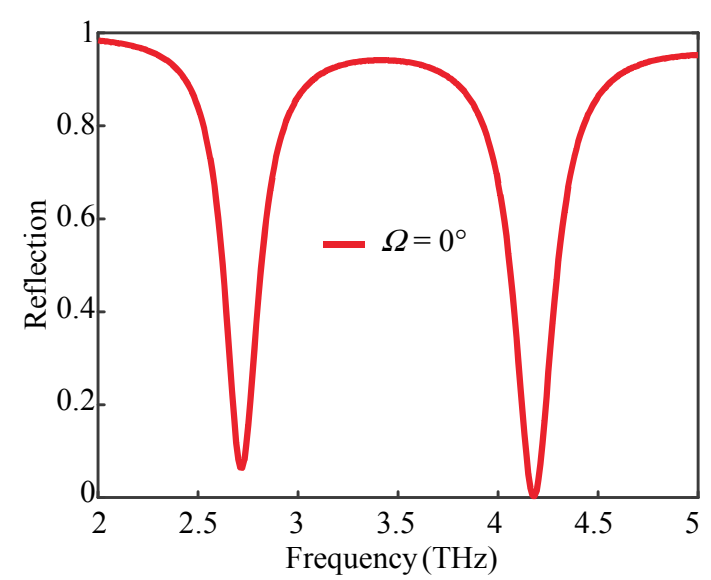

Fig. 3 Reflection $S_{11}^{2}$ in a terahertz range of $2 \mathrm{THz}-5 \mathrm{THz}$. The angle of incidence $\Omega$ of EM plane wave is $0^{\circ}$.

To evaluate the performance of the proposed metamaterial absorber under an incidence EM field $\mathbf{E}$, both the intensity $|\mathbf{E}|^{2}$ and the Poynting vectors or energy flux are analyzed in the near surroundings of the structure. Intensity and Poynting vectors (white arrows) in Fig. 4(a) are plotted for the resonance frequency at $2.71 \mathrm{THz}$ whereas Fig. 4(b) represents the results at $4.17 \mathrm{THz}$. The field $\mathbf{E}$ is polarized along the $y$-axis (TE mode). Most of the energy is accumulated in the gap along the $x$-axis between upper and lower pairs of RAEs, which is indicated by the pink colored ellipse in the figure. It is interesting to notice the direction of flow of energy as depicted by the Poynting vectors on the metallic RAEs. The length of arrows represents the magnitude of the energy flux. The longer arrows are settled in the gap (in the center) along the $x$-axis. The phenomenon indicates a general behavior of the resonator wherein a huge amount of energy is stored in the tiny spaces. However, it is noticeable in Fig. 4(b) that the magnitude of the Poynting vectors is lower than that in Fig. 4(a). The reason could be that at $4.17 \mathrm{THz}$, most of the energy is accumulated in the gap rather than those in the metallic parts. In Figs. 4(c) and 4(d), current densities are plotted for $2.71 \mathrm{THz}$ and $4.17 \mathrm{THz}$, respectively. Small white arrows represent the nature of charges, i.e., positive or negative. It is observed that for the TE mode of a plane wave, almost all charges are settled inside the gap along the $x$-axis (white color ellipse). However, in Fig. 4(d) at $4.17 \mathrm{THz}$, charges are gathered fairly in the central part. Furthermore, it is interesting to notice from the direction of the arrows that charges are arranged in the opposite direction in Figs. 4(c) and 4(d), e.g., arrows point upward for $2.71 \mathrm{THz}$ and downward for $4.17 \mathrm{THz}$. Similar results can be observed for a TM mode where the Poynting vectors and the current density are arranged along the $y$-axis direction. In general, the structure behaves uniformly well under the influence of $x$ or $y$ polarized incident EM plane wave. This impressive performance is a manifestation of the structure's insensitivity to both TE and TM modes. The right-angle shaped elements enhance the charge storing ability of the structure by aligning the charges in the direction of the field, which eventually induces a high absorption within the structure. The design of the structure is simple, the micro-gaps between the RAEs which gather the charges, are aligned only along the vertical and horizontal directions [46, 47] and hence are relatively easy to be manufactured in practice contrast to the complicated structures (e.g., round shaped microfeatures).

A perfect absorber provides $100 \%$ absorbance with respect to any angle of incidence and state of polarization of an EM wave [10]. However, minor fluctuations in absorbance and frequency usually occur in a typical absorber. Therefore, a good absorber must provide a relatively high absorbance with the minimum variations [35]. Herein, we calculate the absorbance for different incidence angles for TE and TM modes. The results are shown in Fig. 5. In Fig. 5(a), the incidence field is polarized along the $y$-axis, and in Fig. 5(b), the field is polarized along the $x$-axis. In both cases, the range of angles $\Omega$ is $0-70^{\circ}$. The angle $\Omega$ is defined as an angle between the wave vector $\mathbf{k}$ and the normal to 

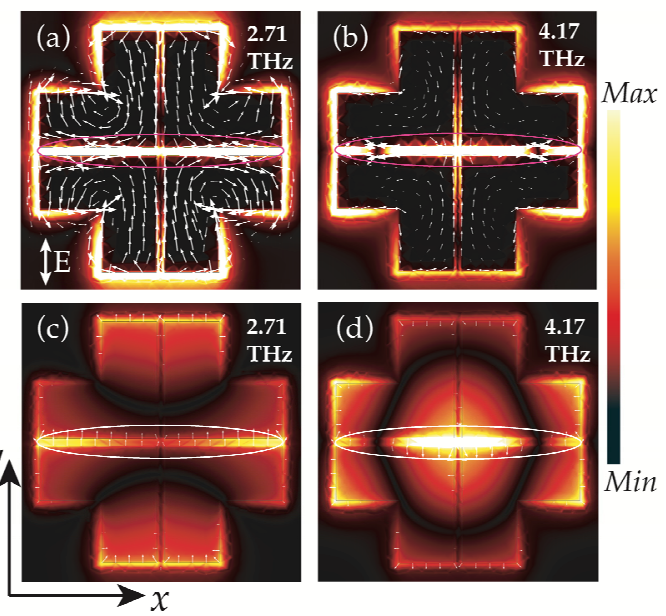

Fig. 4 Distribution of Poynting vectors (white arrows) in (a) and (b) in near surroundings of the structure. In (c) and (d), current density (white arrows) is plotted. The color bar represents the intensity and charge distribution magnitude. Only the TE case is considered.

the surface in the plane of incidence. At resonant frequencies of $2.71 \mathrm{THz}$ and $4.17 \mathrm{THz}$, two absorbance peaks are noticed. For the TE mode at $2.71 \mathrm{THz}$, the absorbance value is $\sim 90 \%$ for all the angles of incidence. A slight change in the absorbance is observed as a consequence of impedance mismatch, which will be discussed into details later. At the resonant frequency of $4.17 \mathrm{THz}$, the absorbance achieves a maximum value of $100 \%$ at angles $0^{\circ}-40^{\circ}$. However, the absorbance value drops to $\sim 80 \%$ at $\Omega=70^{\circ}$. In Fig. 5(b), for the TM case, the absorbance approaches $100 \%$ at $2.71 \mathrm{THz}$ for $\Omega=60^{\circ}$ and $70^{\circ}$. The overall absorbance is more than $90 \%$ for $\Omega=0^{\circ}-70^{\circ}$. Also, the absorbance is $\sim 90 \%$ for all angles of incidence at $4.17 \mathrm{THz}$. Therefore, in general, the absorber is insensitive to a wide range of incidence angles $\Omega=0^{\circ}-70^{\circ}$. In Figs. 5(a) and 5(b), we observe a slight shift in frequency, however, a maximum shift from $2.71 \mathrm{THz}$ at $70^{\circ}$ is comparable with the spectral resolution (c.a. $0.03 \mathrm{THz}$ or $1 \mathrm{~cm}^{-1}$ ) of a typical terahertz time domain spectrometer [48]. The right-angle shaped elements enhance the charge storing ability of the structure by aligning density of charges in the direction of the field, hence inducing a high absorption within the structure (see Fig.4). In other words, almost all the field entering the structure is used in the driving and perfectly alignment of charges within the structure. In addition, the electric and magnetic fields at the selected wide range of angles remain almost unchanged due to the symmetric nature of the device, which illustrates the physical mechanism of the observed high absorbance by the proposed metamaterial device. Further evidence of the mechanism is verified by Fig. 5 wherein the absorbance is high for a range of incidence angles for either TE or TM modes.

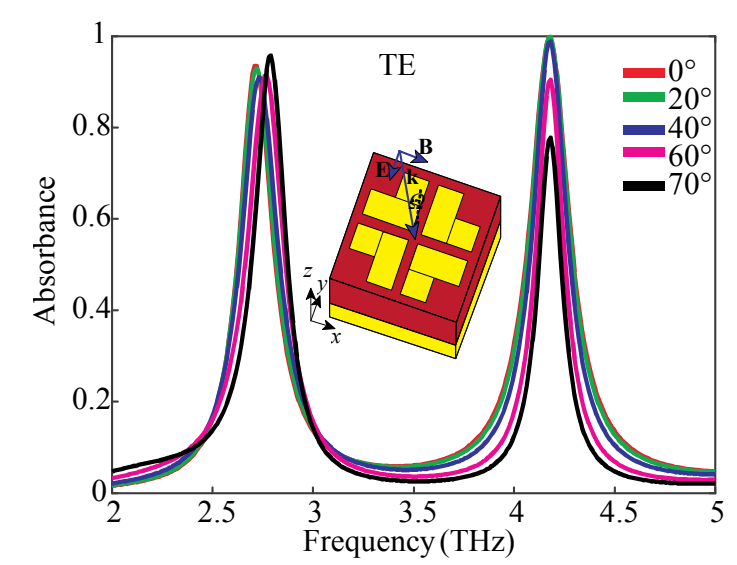

(a)

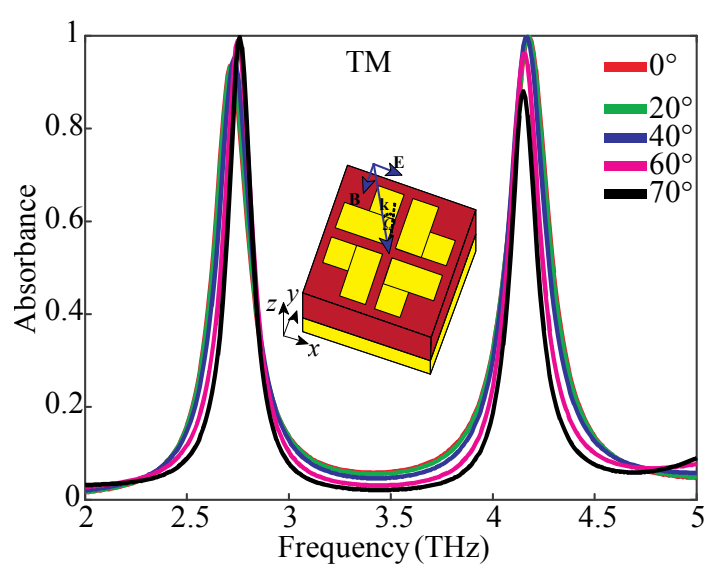

(b)

Fig. 5 Absorbance for an incidence range of angle $0^{\circ}-70^{\circ}$. In Fig. 5(a), absorbance is plotted for a TE mode of an incidence EM wave, and in Fig. 5(b), absorbance is plotted for a TM mode. In both cases, the incidence field $\mathbf{E}$ is demonstrated inside the figures.

For a perfect absorber at a resonant frequency, real and imaginary parts of impedance should 
approach 1 and 0 , respectively. However, due to the change in the angle of incidence with respect to the structure, the variation in absorbance appears that corresponds to the impendence mismatch. To understand this occurrence of change in absorbance, the real and the imaginary components of the relative impendence $Z$ are calculated. The results, only for the TM mode at $2.71 \mathrm{THz}$, are shown in Fig. 6. The real part (red circles) approaches 1 (black color dotted horizontal line) at large incidence angles of EM wave, e.g., $60^{\circ}$ and $70^{\circ}$. Likewise, the imaginary part (green circles) approaches zero towards large angles. This behavior of impedance indicates that the absorbance should be maximum at $60^{\circ}$ and $70^{\circ}$, which indeed is true as shown in Fig. 5(b). Therefore, the observed change in the absorbance as a function of an angle of incidence is due to the occurrence of impendence mismatch.

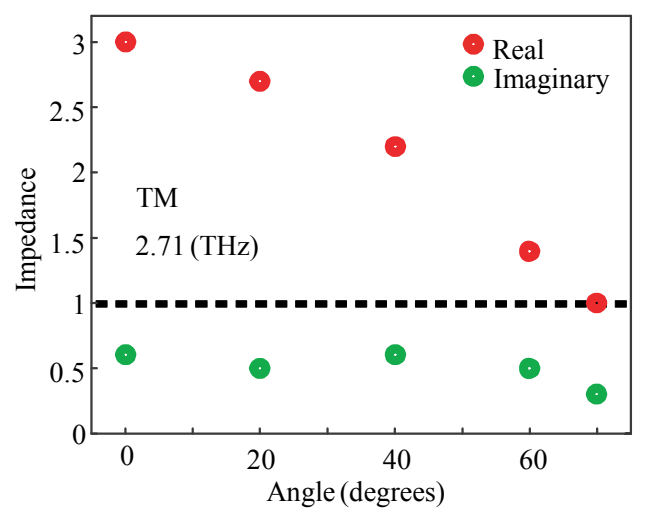

Fig. 6 Real and imaginary parts of relative impedance as a function of an angle of incidence for a TM mode at $2.71 \mathrm{THz}$.

To investigate the performance of the metamaterial absorber for different states of polarization of the incidence EM wave, we calculate the absorbance as a function of polarization angle $\alpha$. The results are presented in Fig. 7. $\alpha$ is defined as an angle between the electric field $\mathbf{E}$ and $x$-axis, and it is demonstrated by the insert of Fig. 7. Due to the symmetric nature of the structure, the absorbance is calculated only for a range of $\alpha=0^{\circ}-90^{\circ}$ at an incidence angle of $\Omega=0^{\circ}$. The absorbance is more than $80 \%$ for all polarization angles at $2.71 \mathrm{THz}$. Nevertheless, a clear variation in the resonant frequency $(\mathrm{THz})$ can be seen for different values of $\alpha$, which will be discussed in the next section. The absorbance is $100 \%$ at $4.17 \mathrm{THz}$ for $\alpha=0^{\circ}-90^{\circ}$ which indicates that the absorber, in terms of absorbance, is independent for any state of polarization of the incidence EM wave.

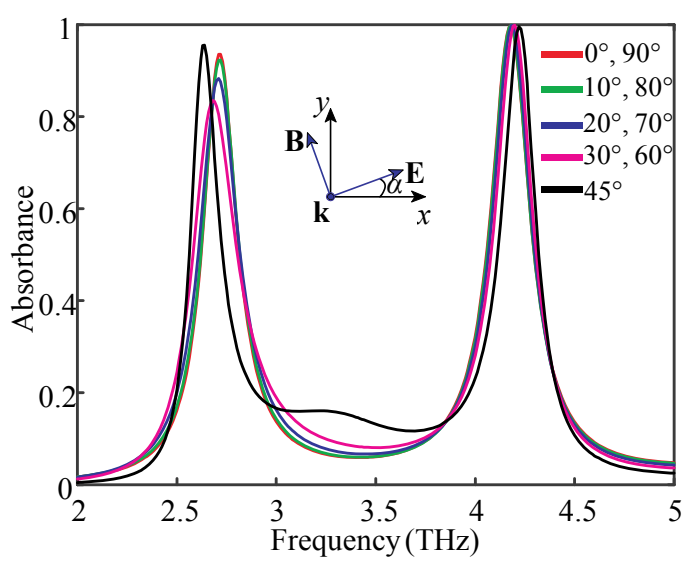

Fig. 7 Absorbance for different polarization angles of $\alpha=$ $0^{\circ}-90^{\circ}$. Inside the figure, the angle $\alpha$ is demonstrated.

To analyze the observed shift in the resonant frequency in Fig. 7 for different polarization angles, we plot the frequency shifts of the absorbance peaks as a function of $\alpha$. The results are shown in Fig. 8 for both resonant frequencies, $2.71 \mathrm{THz}$ and $4.17 \mathrm{THz}$. In Fig. 8(a), we observe a maximum shift of $0.08 \mathrm{THz}$ at $\alpha=45^{\circ}$. It is interesting to notice the behavior of the frequency shift from the resonant frequency. For example, the shift decreases as $\alpha$ increases from $0^{\circ}$ to $45^{\circ}$ and begins to increase at angles greater than $45^{\circ}$ until it reaches a maximum value of $2.70 \mathrm{THz}$ at $90^{\circ}$, which is almost equal to the resonant frequency $2.71 \mathrm{THz}$. This behavior of the frequency shift as a function of $\alpha$ emanates from the symmetric nature of the proposed structure consists of RAEs. To make this trend more visible, a fitted curve is plotted as well. A maximum shift of $0.04 \mathrm{THz}$ from the resonant frequency of $4.17 \mathrm{THz}$ at $\alpha=45^{\circ}$ is observed in Fig. 8(b). Unlike Fig. 8(a), the frequency shift increases till $45^{\circ}$ and afterwards deceases back to $4.17 \mathrm{THz}$ at $90^{\circ}$, which again serves as an evident of the symmetric nature of the resonant absorber. 
Despite the observed shift in frequency, the general response of the absorber in terms of absorbance is relatively high $(80 \%)$ for any angle of polarization $\alpha$.

The manufacturing of the sample is not a part of this work; however, the proposed design is simple with size in the micrometer scale, which makes its fabrication relatively easy. In addition, due to the commercial availability of the used materials (A1 and $\mathrm{TiO}_{2}$ ), it is possible to fabricate the metamaterial absorber by considering commonly applied nanofabrication techniques. For example, layers of $\mathrm{Al}$ and $\mathrm{TiO}_{2}$ [49] could be deposited by thermal evaporation and atomic layer deposition methods. The RAEs of $\mathrm{Al}$ at the top are achievable by employing the nanolithography technique. The structure could be built on often used substrate, e.g., silicon.

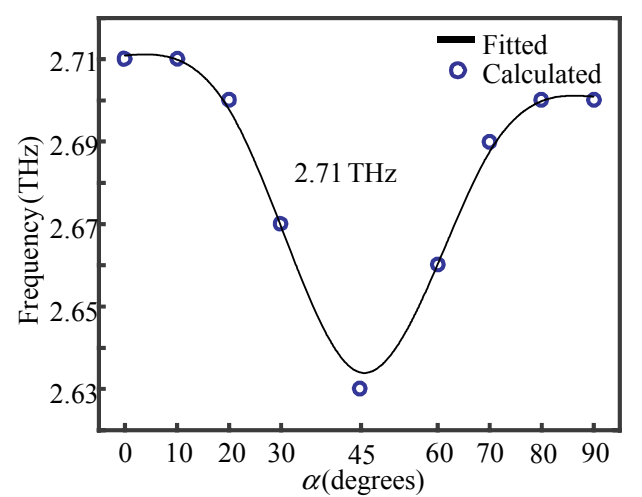

(a)

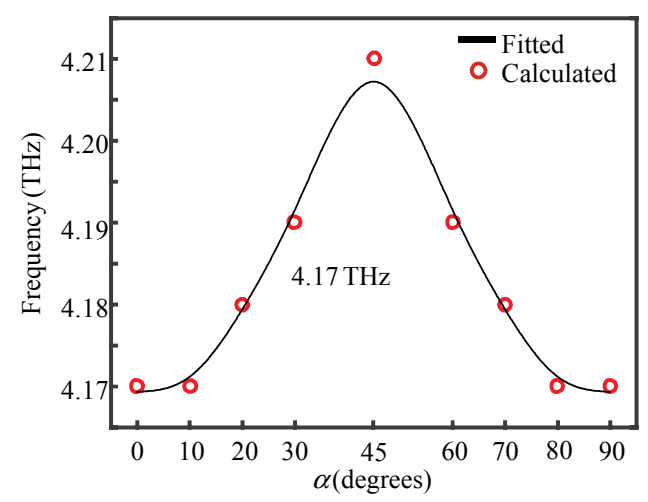

(b)

Fig. 8 Shift in frequency as a function of polarization angle $\alpha$ : (a) shift is plotted for a resonant frequency $2.71 \mathrm{THz}$ while in (b) shift is plotted for $4.17 \mathrm{THz}$. To make the behavior of shifts more visible, a fitted curve is plotted as well.

\section{Conclusions}

In summary, we propose and analyze a dual-band metamaterial absorber in a terahertz region by numerical simulations. The absorber is insensitive to a wide range of incidence angles $0^{\circ}-70^{\circ}$ and keeps high absorption value $\sim 80 \%$ for a TE and a TM mode of an incidence EM wave. Furthermore, the absorber due to its symmetric nature shows an absorbance that is more than $80 \%$ for all angles of polarization of a plane wave. We discuss the origin of variations in absorbance and frequency as a function of incidence and polarization angles. The proposed structure is simple in design and has a size in micrometers. Therefore, we present a possible fabrication route of manufacturing a device due to commercial availability of $\mathrm{Al}$ and $\mathrm{TiO}_{2}$.

Open Access This article is distributed under the terms of the Creative Commons Attribution 4.0 International License (http://creativecommons.org/licenses/by/4.0/), which permits unrestricted use, distribution, and reproduction in any medium, provided you give appropriate credit to the original author(s) and the source, provide a link to the Creative Commons license, and indicate if changes were made.

\section{References}

[1] E. Shamonina and L. Solymar, "Metamaterials: how the subject started," Metamaterials, 2007, 1(1): 12-18.

[2] D. R. Smith, "Metamaterials and negative refractive index," Science, 2004, 305(5685): 788-792.

[3] J. Kim, K. Han, and J. W. Hahn, "Selective dual-band metamaterial perfect absorber for infrared stealth technology," Scientific Reports, 2017, 7(1): 6740.

[4] V. M. Shalaev, "Optical negative-index metamaterials," Nature Photonics, 2007, 1(1): 41-48.

[5] A. M. Fox, Optical properties of solids. Oxford: Oxford University Press, 2001.

[6] J. B. Pendry, "Negative refraction makes a perfect lens," Physical Review Letters, 2000, 85(18): 3966-3969.

[7] C. Wu, I. N. Burton, G. Shvets, J. John, A. Milder, B. Zollars, et al., "Large-area wide-angle spectrally 
selective plasmonic absorber," Physical Review B, 2011, 84(7): 075102.

[8] T. Cao, C. Wei, R. E. Simpson, L. Zhang, and M. J. Cryan, "Broadband polarization-independent perfect absorber using a phase-change metamaterial at visible frequencies," Scientific Reports, 2014, 4: 3955.

[9] Y. Cheng, H. Yang, Z. Cheng, and N. Wu, "Perfect metamaterial absorber based on a split-ring-cross resonator," Applied Physics A, 2011, 102(1): 99-103.

[10] N. I. Landy, S. Sajuyigbe, J. J. Mock, D. R. Smith, and W. J. Padilla, "Perfect metamaterial absorber," Physical Review Letters, 2008, 100(20): 207402.

[11] H. Tao, C. M. Bingham, A. C. Strikwerda, D. Pilon, D. Shrekenhamer, N. I. Landy, et al., "Highly flexible wide angle of incidence terahertz metamaterial absorber: design, fabrication, and characterization," Physical Review B, 2008, 78(24): 241103.

[12] K. Aydin, V. E. Ferry, R. M. Briggs, and H. A. Atwater, "Broadband polarization-independent resonant light absorption using ultrathin plasmonic super absorbers," Nature Communications, 2011, 2: 517.

[13] W. Li, X. Zhou, Y. Ying, X. Qiao, F. Qin, Q. Li, et al." Polarization-insensitive wide-angle multiband metamaterial absorber with a double-layer modified electric ring resonator array," AIP Advances, 2015, 5(6): 067151.

[14] D. Lim, D. Lee, and S. Lim, "Angle- and polarization-insensitive metamaterial absorber using via array," Scientific Reports, 2016, 6(1): 39686.

[15] Y. Q. Ye, Y. Jin, and S. He, "Omnidirectional, polarization-insensitive and broadband thin absorber in the terahertz regime," Journal of the Optical Society of America B, 2010, 27(3): 498.

[16] L. Cong, S. Tan, R. Yahiaoui, F. Yan, W. Zhang, and R. Singh, "Experimental demonstration of ultrasensitive sensing with terahertz metamaterial absorbers: a comparison with the metasurfaces," Applied Physics Letters, 2015, 106(3): 031107.

[17]H. Tao, N. I. Landy, C. M. Bingham, X. Zhang, R. D. Averitt, and W. J. Padilla, "A metamaterial absorber for the terahertz regime: design, fabrication and characterization," Optics Express, 2008, 16(10): 7181.

[18] T. T. Nguyen and S. Lim, "Wide incidence angle-insensitive metamaterial absorber for both TE and TM polarization using eight-circular-sector," Scientific Reports, 2017, 7(1): 3204.

[19] T. Wu, J. Lai, S. Wang, X. Li, and Y. Huang, "UV-visible broadband wide-angle polarization-insensitive absorber based on metal groove structures with multiple depths," Applied Optics, 2017, 56(21): 5844.
[20] N. T. Trung, D. Lee, H. K. Sung, and S. Lim, "Angle- and polarization-insensitive metamaterial absorber based on vertical and horizontal symmetric slotted sectors," Applied Optics, 2016, 55(29): 8301.

[21] S. Shang, S. Yang, L. Tao, L. Yang, and H. Cao, "Ultrathin triple-band polarization-insensitive wide-angle compact metamaterial absorber," AIP Advances, 2016, 6(7): 075203.

[22] X. J. He, Y. Wang, J. Wang, T. Gui, and Q. Wu, "Dual-band terahertz metamaterial absorber with polarization insensitivity and wide inciden angle," Progress in Electromagnetics Research, 2011, 115: 381-397.

[23] Y. Ma, Q. Chen, J. Grant, S. C. Saha, A. Khalid, and D. R. S. Cumming, "A terahertz polarization insensitive dual band metamaterial absorber," Optics Letters, 2011, 36(6): 945.

[24] Q. Y. Wen, H. W. Zhang, Y. S. Xie, Q. H. Yang, and Y. L. Liu, "Dual band terahertz metamaterial absorber: design, fabrication, and characterization," Applied Physics Letters, 2009, 95(24): 241111.

[25] X. Huang, C. Lu, C. Rong, and M. Liu, "Wide-angle perfect metamaterial absorbers based on cave-rings and the complementary patterns," Optical Materials Express, 2018, 8(9): 2520.

[26] X. Huang, C. Lu, C. Rong, Z. Hu, and M. Liu, "Multiband ultrathin polarization-insensitive terahertz perfect absorbers with complementary metamaterial and resonator based on high-order electric and magnetic resonances," IEEE Photonics Journal, 2018, 10(6): 1-11.

[27] T. Cao, S. Wang, and C. W. Wei, "Simulation of tunable metamaterial perfect absorber by modulating $\mathrm{Bi}_{2} \mathrm{Se}_{3}$ dielectric function," Materials Express, 2016, 6(1): 45-52.

[28] W. Dong, Y. Qiu, J. Yang, R. E. Simpson, and T. Cao, "Wideband absorbers in the visible with ultrathin plasmonic-phase change material nanogratings," The Journal of Physical Chemistry C, 2016, 120(23): 12713-12722.

[29] T. Cao, L. Zhang, R. E. Simpson, and M. J. Cryan, "Mid-infrared tunable polarization-independent perfect absorber using a phase-change metamaterial," Journal of the Optical Society of America B, 2013, 30(6): 1580.

[30] T. Cao, R. E. Simpson, and M. J. Cryan, "Study of tunable negative index metamaterials based on phase-change materials," Journal of the Optical Society of America B, 2013, 30(2): 439.

[31] T. Cao, C. Wei, R. E. Simpson, L. Zhang, and M. J. Cryan, "Rapid phase transition of a phase-change metamaterial perfect absorber," Optical Materials Express, 2013, 3(8): 1101.

[32] G. P. Williams, "Filling the THz gap-high power sources and applications," Reports on Progress in Physics, 2005, 69(2): 301-326.

[33] C. L. Holloway, E. F. Kuester, J. A. Gordon, J. 
O'Hara, J. Booth, and D. R. Smith, “An overview of the theory and applications of metasurfaces: the two-dimensional equivalents of metamaterials," IEEE Antennas and Propagation Magazine, 2012, 54(2): 10-35.

[34] Y. J. Yoo, J. S. Hwang, and Y. P. Lee, "Flexible perfect metamaterial absorbers for electromagnetic wave," Journal of Electromagnetic Waves and Applications, 2017, 31(7): 663-715.

[35] C. Gong, M. Zhan, J. Yang, Z. Wang, H. Liu, Y. Zhao, et al., "Broadband terahertz metamaterial absorber based on sectional asymmetric structures," Scientific Reports, 2016, 6(1): 32466.

[36] X. Liu, C. Lan, B. Li, Q. Zhao, and J. Zhou, "Dual band metamaterial perfect absorber based on artificial dielectric 'molecules'," Scientific Reports, 2016, 6(1): 28906.

[37] X. Liu, C. Lan, K. Bi, B. Li, Q. Zhao, and J. Zhou, "Dual band metamaterial perfect absorber based on Mie resonances," Applied Physics Letters, 2016, 109(6): 062902.

[38] B. Zhang, Y. Zhao, Q. Hao, B. Kiraly, I. Khoo, S. Chen, et al.,"Polarization-independent dual-band infrared perfect absorber based on a metal-dielectric-metal elliptical nanodisk array," Optics Express, 2011, 19(16): 15221.

[39] H. M. Lee and H. Lee, "A dual-band metamaterial absorber based with resonant-magnetic structures," Progress in Electromagnetics Research, 2012, 33: $1-12$.

[40] Y. Ma, H. Zhang, Y. Li, and Y. Wang, "Miniaturized and dual-band metamaterial absorber with fractal Sierpinski structure," Journal of the Optical Society of America B, 2014, 31(2): 325.

[41] K. Z. Rajab, M. Naftaly, E. H. Linfield, J. C. Nino, D. Arenas, D. Tanner, et al. "Broadband dielectric characterization of aluminum oxide $\left(\mathrm{Al}_{2} \mathrm{O}_{3}\right)$," Journal of Microelectronics and Electronic
Packaging, 2008, 5(1): 2-7.

[42] N. Matsumoto, T. Hosokura, K. Kageyama, H. Takagi, Y. Sakabe, and M. Hangyo, "Analysis of dielectric response of $\mathrm{TiO}_{2}$ in terahertz frequency region by general harmonic oscillator model," Japanese Journal of Applied Physics, 2008, 47(9): 7725-7728.

[43] A. D. Rakić, A. B. Djurišić, J. M. Elazar, and M. L. Majewski, "Optical properties of metallic films for vertical-cavity optoelectronic devices," Applied Optics, 1998, 37(22): 5271.

[44] J. R. DeVore, "Refractive indices of rutile and sphalerite," Journal of the Optical Society of America, 1951, 41(6): 416.

[45] D. M. Pozar, Microwave engineering, 4th Ed. USA: Wiley, 2011.

[46] S. Daniel and P. Bawuah, "Highly polarization and wide-angle insensitive metamaterial absorber for terahertz applications," Optical Materials, 2018, 84: 447-452.

[47] Y. Bai, L. Zhao, D. Ju, Y. Jiang, and L. Liu, "Wide-angle, polarization-independent and dual-band infrared perfect absorber based on L-shaped metamaterial," Optics Express, 2015, 23(7): 8670.

[48] E. P. J. Parrott, J. A. Zeitler, T. Friščić, M. Pepper, W. Jones, G. M. Day, et al., "Testing the sensitivity of terahertz spectroscopy to changes in molecular and supramolecular structure: a study of structurally similar cocrystals," Crystal Growth and Design, 2009, 9(3): 1452-1460.

[49] N. Negishi, S. Matsuzawa, K. Takeuchi, and P. Pichat, "Transparent micrometer-thick $\mathrm{TiO}_{2}$ films on $\mathrm{SiO}_{2}$-coated glass prepared by repeated dip-coating/calcination: characteristics and photocatalytic activities for removing acetaldehyde or toluene in air," Chemistry of Materials, 2007, 19(15): 3808-3814. 